\title{
EFFECT OF DIFFERENT MINI IMPLANTS DISTRIBUTION FOR MANDIBULAR OVERDENTURE CASES ON MAXILLARY RIDGE HEIGHT. RETROSPECTIVE RADIOGRAPHIC STUDY
}

\author{
Ahmad Mohmad Elrawdy*, Kawkb Mohmad Eltamimi** and Mohammed Ezzat El Sayed ${ }^{* * *}$
}

\begin{abstract}
Statement of problem. Mandibular overdentures assisted by mini implants induce sever bone loss of anterior maxilla.

Purpose. The purpose of the study was to evaluate the maxillary ridge change under complete dentures opposed by mandibular overdentures assisted by four mini-implants, in case of interforaminal distribution or in case of wide distribution after wearing dentures for 6 years.

Subjects and methods. The subjects enrolled for this study $(n=20)$. They were treated with maxillary complete dentures, randomly assigned into two equal groups: Group I received mandibular overdenture assisted by four mini implants distributed equally in the inter-foraminal region exposed to immediate loading protocol. Group II received mandibular overdenture assisted by four mini-implants (two inserted in canine region and two inserted in first molar region) exposed to immediate loading protocol. Radiographic evaluation of maxillary bone height using digital panorama for every patient of both groups was recorded at the time of mini-implants insertion and after 6 years.
\end{abstract}

Results: The results showed non- significant differences using independent t-test between wide and inter-foraminal distribution groups for midline, premaxilla. While, for maxillary tuberosity, the results gave significant difference between wide and inter-foraminal distributions at significant levels $\mathrm{P}<0.05$. According to correlation coefficients of bone changes between premaxilla \& maxillary tuberosity at wide and inter-foraminal distribution there were negative correlation for the wide distribution and highly negative correlation in the inter-foraminal distribution.

Conclusion: The results of the present study could confirm the suggestion that the combination syndrome also may occur in conventional maxillary dentures opposed by mini implants assisted mandibular overdentures and wide mini implants distribution has similar effect as inter-foraminal distribution in the maxillary alveolar ridge resorption.

KEYWORDS: Inter-foraminal mini-implants distribution, wide mini-implants distribution, mini-implants assisted overdenture, maxillary ridge height changes, digital panoramic radiographic evaluation.

\footnotetext{
* Assistant Professor,Oral Radiology Department,Faculty of Dentistry,Suez Canal University

** Lecturer, Prosthodontics Department, Faculty of Dentistry, Suez Canal University

*** Assistant Professor, Removable Prosthodontics Department , Faculty of Dentistry Suez Canal University
} 


\section{INTRODUCTION}

Residual ridge resorption (RRR) in patients wearing complete dentures is a continuous, unavoidable process affected by the type of prosthesis that cover and oppose the residual ridge, which compromise the retention and stability especially in mandibular cases ${ }^{(1,2)}$. Osseointergrated implants retained overdentures have been used for rehabilitation of edentulous mandible is cost-effective and fulfill the satisfaction of patients. ${ }^{(3)}$

Mini implants may be the best choice of treatment for patients with inadequate ridge width, which does not allow the placement of conventional sized implants without further surgical procedures. ${ }^{(4-7)}$ Four mini-implants at the inter-foraminal region have been exposed for immediate loading with mandibular overdentures. These mini-implants were able to improve the mandibular overdenture's retention, the patient's life quality, the patient satisfaction, and the chewing ability of the overdenture wearers. ${ }^{(4,6)}$

Most researches have mainly centered on the effect of treatment on the mandible, while only a few researches have centered on the residual ridge resorption in the maxilla. ${ }^{(8-12)}$ In previous studies, it was reported that the chance of developing the combination syndrome in persons wearing mandibular implant-retained overdentures. The five symptoms that commonly occur in the combination syndrome are (i) loss of bone from the anterior part of the maxillary ridge, (ii) development of fibrous or bony enlargements of the tuberosities, (iii) papillary hyperplasia of the hard palate, (iv) extrusion of the mandibular anterior teeth and (v) reduction in posterior mandibular bone ${ }^{(8,11,13)}$. Saunders et al (14), have proposed that the combination syndrome begins with residual ridge resorption of the mandibular posterior areas. This resorption progressively causes loss of posterior occlusal load and increase in anterior occlusal load. This increased anterior loading may produce resorption of the residual ridge of the maxillary anterior area. As the anterior maxilla continue resorption, it will create space posteriorly which induces the posterior maxilla enlargement with downward growth of the tuberosities. ${ }^{(15,16)}$

On the other hand, other studies found that no difference occur in the rate of maxillary residual ridge resorption between the mandibular implants retained over dentures and complete dentures or an even more residual ridge resorption in the case of conventional complete denture therapy caused by insufficient retention and stability. ${ }^{(12,17,18)}$

Panoramic radiographs have become routine diagnostic tool for treatment planning of edentulous patients. Atwood did an assessment of the maxillary ridge as it was never done before; it was done by measuring the constant landmarks of the maxilla and then deriving the ratios of anterior and posterior bone resorption. ${ }^{(19)}$

We pose the following question because of this situation: Is the wide distribution of mini-implants, in mandibular arch improves maxillary ridge resorption?

\section{SUBJECTS AND METHODS}

Twenty male patients had completely edentulous ridges were selected from the Outpatients Clinic, Faculty of Dentistry, Suez Canal University. The study protocol was explained in detail to all patients and their consents for participating in the study were taken.

\section{Patient selection}

The selected patients had the following criteria: Free from any systemic diseases detected by blood / urine analysis that may affect bone quality and quantity. Their ages ranged from 50 to 60 years old with adequate inter-arch space, had a good oral hygiene, Angle's Class I jaw relationship with adequate height of the residual ridges, no bad habits including heavy smoking, bruxism, clenching or tongue thrusting. 


\section{Dentures insertion}

Upper and lower acrylic complete dentures were delivered to all patients with lingualized occlusal scheme with anterior light contact. The patients were recalled for necessary adjustments.

\section{Patients grouping}

The patients were divided into two equal groups according to mini-implants distribution.

Group (I): Received four mini-implants, distributed equally in the interforminal region and exposed to immediate loading protocol.

Group (II): Received four mini-implants, one mini-implant in the canine region and one miniimplant in the first molar region in each side of the arch and exposed to immediate loading protocol.

\section{Implants insertion}

The finished lower denture duplicated for each patient and processed in transparent acrylic resin radiographic stent. At the proposed site for each mini-implant, holes were drilled through the fitting surface of the stent to accommodate a stainlesssteel ball ( $2 \mathrm{~mm}$ in diameter). The balls were totally submerged into the holes. Panoramic radiograph was made with the radiographic stent in the patient's mouth. The ball's images were used to assess the bone height at the proposed mini-implant sites. According to the flapless surgical technique with a traumatic osteotomy and adjustable torque ratchet, four mini-implants (Dentium Co., Korea) $2.5 \mathrm{~mm}$ in diameter and 10 or $12 \mathrm{~mm}$ in length were used according to bone height. The mini-implant sites in group I were four mini-implants distributed equally in the interforminal area. A minimum of $5 \mathrm{~mm}$ mesiodistal distance was kept between adjacent implants in group I, the mini-implants sites in group II were two mini-implants in cusped area and another two mini-implants placed in the first molar area in each side of the mandible. The implants were inserted by using the single stage flapless surgical approach.

\section{Direct Pick-up Procedure}

As soon as the four mini-implants inserted, undercut areas around the mini-implant heads were covered using sterile orthodontic O-rings. The female metallic housing caps were placed on the miniimplants. The female house surface marked with marker, the lower denture seated in patient's mouth. The areas opposing the housings were marked on the tissue surface of the denture. The marked areas were removed, until a clearance space of about $1-2 \mathrm{~mm}$. was provided around the metallic housings. The maxillary and mandibular dentures were seated into the patient's mouth to verify the complete seating of the lower denture without any interferences, rocking or occlusal discrepancy. Four holes were then created in the lingual flange below the artificial teeth to allow the escapement of excess material.

A special type of self-cured acrylic resin that does not generate heat (Secure Hard Pick-Up Kit, 3M ESPE, USA) was mixed and applied in the dough stage to the relieved areas of the fitting surface of the denture. The mandibular denture was reseated in the patient's mouth. The patient was instructed to close in centric occlusion. After complete polymerization, the denture was removed from the patient's mouth picking up the metal housings. Excess material was trimmed using finishing stone, the mandibular miniimplants retained over-denture was repolished and delivered to the patient, and the denture was placed in immediate functional loading on the same day of mini-implants placement.

\section{Radiographic evaluation}

The patients were subjected to two consecutive direct digital panoramic radiographs, initial record after completing implants insertion (T0), and the final record after 6-years post-insertion (T6) respectively. The panoramic images were performed using digital panoramic machine (Sirona, ORTHOPHOS XG5 Ds/ Ceph., Germany) with CCD sensor technology and $27 \mu \mathrm{m}$ pixel size. The exposure parameters for 
all patients were $64 \mathrm{KVp}, 8 \mathrm{Ma}$, and 14.1 sec exposure time. All images were captured, analyzed processed and stored using SIDEXIS XG software (SIDEXIS XG image processing software, Sirona Dental System, Germany). The patients were positioned according to the standard procedure described by Stuart and Michael ${ }^{(20)}$, as follows:

- The patient head was carefully aligned in the focal trough so both arches are in the middle of the focal trough.

- We removed the bite block, instead we placed the metal bar in such case the arch facing toward the column.

- The upper and lower arches are in line by using cotton pellet between them.

- The midsagittal plane was adjusted within the exact of focal trough and perpendicular to the floor.

- The Frankfort plane was aligned parallel with the floor.

- The patient's back and spine was adjusted to be erect with extended neck position.

Both radiographs were performed with the same digital panoramic machine and, as such, the degree of magnification is similar in all radiographs if the patient positioning technique is the same.
Considering that our objective is to find out the difference, the magnification is calculated with the help of the radiographic image of the mini implants.

A line joined the inferior margins of the zygomatic process of the maxilla (Lz). Seven sites were measured on each radiograph first region was at the midline and was determined by images of the nasal septum, anterior nasal spine and nasopalatine foramen (Fig. 1), second, third and fourth regions were consecutively located $10 \mathrm{~mm}$ further distally. The distance from $30 \mathrm{~mm}$ right to $30 \mathrm{~mm}$ left represent premaxilla and the distance from $40 \mathrm{~mm}$ to $70 \mathrm{~mm}$ represent the maxillary tuberosities. The difference between height of alveolar ridge at zero and six years of each distribution was calculated. The positive values indicate bone resorption and negative values indicate bone deposition.

\section{Statistical analysis}

All data was calculated, tabulated and statistically analyzed using suitable statistical tests as follow. A normality test was done to check normal distribution of the sample, and all groups. Statistical analysis was performed using the computer program SPSS software for windows version 22.0 (Statistical Package for Social Science, Armonk, NY: IBM Corp) at significant levels 0.05 (P- Value $\leq 0.5$ ).

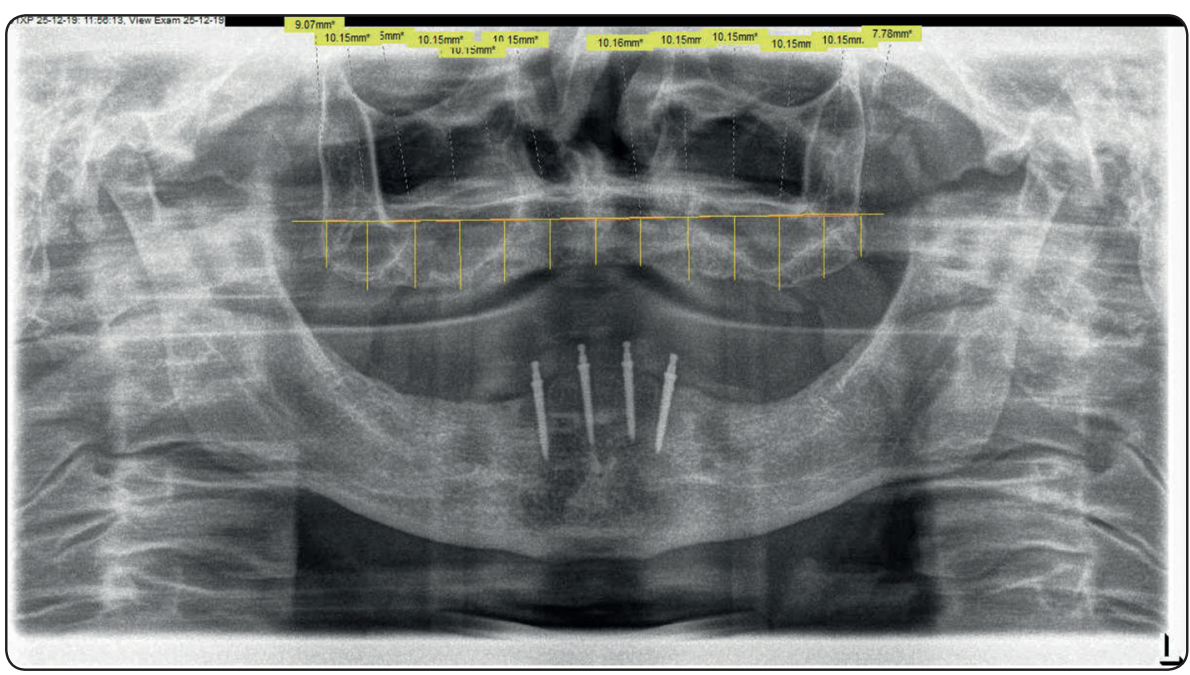

Fig. (1): Digital panoramic radiograph for interforaminal group showing bone height measurements of the maxillary ridge. 


\section{a) Descriptive data}

Descriptive statistics was calculated in the form of Mean \pm Standard deviation (SD), range (Max- Min), median, Coefficient of variance (C.V \%) .... etc.

\section{b) T-test}

Independent T-test was performed for comparison of the mean differences between the two groups at the same time.

\section{RESULTS}

The results showed non-significant differences using independent t-test between wide \& inter-fo- raminal distribution groups for midline, premaxilla with positive mean values $(4.04 \pm 2.02$ and $3.51 \pm 0.35)$ and $(3.40 \pm 1.05,3.46 \pm 0.024)$, respectively. While, for maxillary tuberosity, the results give significant difference between wide $\&$ inter-foraminal distributions with negative values $(-2.10 \pm 0.78,-1.51 \pm 0.002)$ respectively, at significant levels $\mathrm{P}<0.05$. According to correlation coefficients of bone changes between premaxilla \& maxillary tuberosity at wide and inter-foraminal distribution there are negative correlation for the wide distribution (-0.52) and highly negative correlation $(-0.961)$ in the inter-foraminal distribution.

TABLE (1) Show the difference between wide and inter-foraminal distributions at midline and premaxilla $(30-30 \mathrm{~mm})$ and maxillary tuberosity $(40-70 \mathrm{~mm})$ and correlation between bone changes in premaxilla and maxillary tuberosity in both distributions.

\begin{tabular}{|l|c|c|c|c|}
\hline & Midline & Premaxilla & Maxillary tuberosity & \multirow{2}{*}{ Correlation R } \\
\cline { 2 - 5 } & Mean \pm SD & Mean \pm SD & Mean \pm SD & -0.52 \\
\hline Wide distribution & $4.04 \pm 2.02$ & $3.40 \pm 1.05$ & $-2.10 \pm 0.78$ & -0.961 \\
\hline Inter-foraminal distribution & $3.51 \pm 0.35$ & $3.46 \pm 0.024$ & $-1.51 \pm 0.002$ & 0.03 \\
\hline Independent-t-test & 0.87 & 0.98 & $*$ & \\
\hline P- values<0.05 & Ns & Ns & & \\
\hline \multicolumn{2}{|c|}{$*$ ns, means significant and non-significant, respectively. } \\
\hline
\end{tabular}

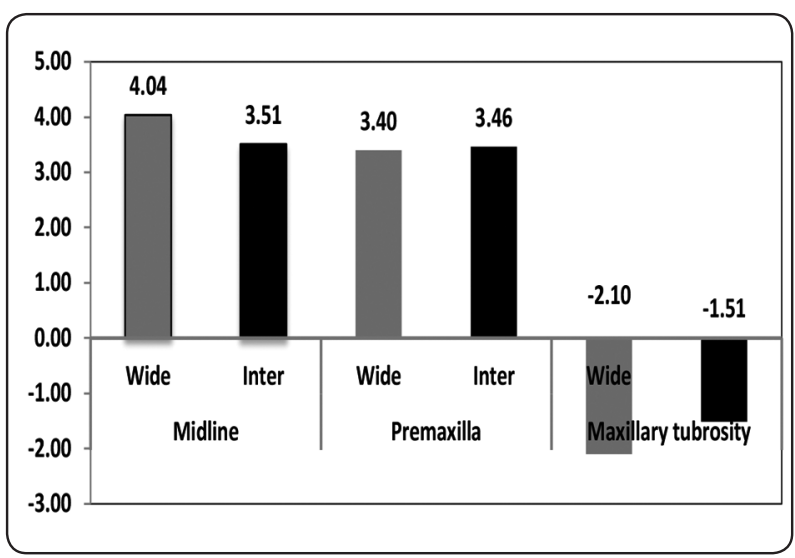

Fig. (2) Show the difference between wide and inter-foraminal distributions at midline, premaxilla and maxillary tuberosity.

\section{DISCUSSION}

Since the use of implants installed in the posterior part of the mandible and their effect on stress distribution in maxillary arch was point of concern in late years the present study aimed to assess the effect of mini implants distributed in mandibular arch on maxillary ridge. Either the mini-implants assisted overdenture assisted by four mini implants installed in the inter-foraminal area or four miniimplants installed in the canine and first molar area in mandibular mini-implants assisted overdenture opposed by maxillary complete denture. 
Panoramic radiographs were the method chosen to assess the bone loss. This type of radiograph is subject to variations in magnification and distortion, although these problems were minimized once we were made aware of the level of magnification of our panoramic radiographs. The magnification is calculated with the help of the radiographic image of the mini implants. This radiographic method is a suitable method for assessing alveolar resorption process. ${ }^{(9,10 \& 21)}$

The bone resorption process that occurred in patients wearing complete dentures was an irreversible phenomenon that occurs in all patients to a greater or lesser degree ${ }^{(22)}$. In this study, bone loss occurs in all the patients, although we do find differences in magnitude from one person to another. This result coincides with that cited by many authors, as well as explaining the multifactorial character of the resorption process. ${ }^{(1,21 \& 22)}$

In our study, we observed bone loss in midline point, there was more bone resorption in the wide distribution group $(0.67 \mathrm{~mm} /$ year $)$ than interforminal group $(0.59 \mathrm{~mm} /$ year $)$ but the difference was not to a significant degree. Lopez-Roldan et al., ${ }^{(23)}$ reported that the midline bone loss in implant supported overdentures is $0.32 \mathrm{~mm} /$ year in comparison with the control group, whose loss at the point indicated was $0.22 \mathrm{~mm} / \mathrm{year}$. The bone loss that Kelly (15) observed in his study regarding the midline was $0.43 \mathrm{~mm} /$ year. Barber ${ }^{(8)}$ observed resorption in the midline, and this occurs at a speed of $0.36 \mathrm{~mm} /$ year.

In premaxilla there was bone loss and it was more at inter-foraminal group than wide distribution group and the difference wasn't statistically significant. The mandibular implants over denture exerted a greater bite force, which may have led to more rotation moment against the opposing complete denture, which, in turn, increased the stress and strain in the buccal and occlusal aspects of the premaxilla. Accordingly, more bone resorption was observed in these regions ${ }^{(2)}$.
In maxillary tuberosity there was increase in bone height in maxillary tuberosities in both groups. The increase in bone height was higher in wide distribution group. Bone loss from the anterior maxilla is responsible for the following consequences. With the anterior bone loss, a flabby hyperplastic tissue will cover the anterior maxilla, which does not bear the denture base and it will flex forward, forming denture fissuratium. As the anterior maxilla continue resorption, it will create space posteriorly which induces the posterior maxilla enlargement with downward growth of the tuberosities. ${ }^{(15,16)}$

There was highly negative correlation between premaxilla \& maxillary tuberosity for the interforaminal distribution and negative correlation for the wide distribution. The results of the present study could confirm the suggestion that the combination syndrome also may occur in conventional maxillary dentures opposed by mini implants assisted mandibular overdentures.

\section{REFERENCES}

1. Alsaggaf A, Fenlon M. A case control study to investigate the effects of denture wear on residual alveolar ridge resorption in edentulous patients. J Dent.2020;98:225-229.

2. Mohamed S, Rohana A, Noor Hayati A, Solehuddin S,Wahyu K. Premaxilla Stress Distribution and Bone Resorption Induced by Implant Overdenture and Conventional Denture. J Prosth 2019;28 :764-770.

3. Emamia E, Cerutti-Kopplin D, Menassa M, Audy N, Kodamac N, Robert Duranda R, Rompréa P, de Grandmont P. Does immediate loading affect clinical and patient-centered outcomes of mandibular 2-unsplinted-implant overdenture? A 2-year within-case analysis. J Dent.2016;50:30-36.

4. Cleidiel L, Fellippo V, de Souza B, Ferreira J, Caroline M et al. Complete overdentures retained by mini implants: A systematic review. J Dent.2017;57:4-13.

5. Shatkin T, Shatkin S, Oppenheimer B, Oppenheimer A. Mini dental implants for long-term fixed and removable prosthetics: a retrospective analysis of 2514 implants placed over a five-year period. Compend Contin Educ. Dent. 2007; 28:92-101. 
6. Preoteasa E, Imre M, Preoteasa CT. A 3-year follow-up study of overdentures retained by mini-dental implants. Int J Oral Maxillofac. Implants 2014; 29:1170-6.

7. Pongsakorn W, Pimduen R, Chaiy R, Pathawee K. Effects of different numbers of mini-dental implants on alveolar ridge strain distribution under mandibular implant-retained overdentures. J. Prosth. Research 2018; 62:35-43.

8. Barber H, Scott R, Maxson B, Fonseca R. Evaluation of anterior maxillary alveolar ridge resorption when opposed by the trans mandibular implant. J. Oral Maxillofac. Surg. 1990; 48:1283-1287.

9. Jacobs R, Van Steenberghe D, Nys M, Naert I. Maxillary bone resorption in patients with mandibular implant-supported overdentures or fixed prostheses. J Prosth. Dent. 1993;70: 135-140.

10. Kreisler M, Behneke N, Behneke A, d'Hoedt B. Residual ridge resorption in the edentulous maxilla in patients with implant-supported mandibular overdentures: an 8-year retrospective study. Int J Prosthodont. 2003; 16:295-300.

11. Gupta R, Luthra P, Sharma A. Combination syndrome-a review. Int J Res Health Allied Sci 2016; 2:41-43.

12. Narhi TO, Geertman ME, Hevinga M, Abdo H, Kalk W. Changes in the edentulous maxilla in persons wearing implant-retained mandibular overdentures. J. Prosth. Dent. 2000;84:43-49.

13. Jacobs R, Schotte A, van SD, Quirynen M, Naert I. Posterior jawbone resorption in Osseointergrated implant supported overdentures. Clin. Oral Implants Res. 1992;3: 63-70.

14. Saunders T, Gillis RE J, Desjardins R. The maxillary complete denture opposing the mandibular bilateral distal extension partial denture: treatment considerations. J. Prosth. Dent. 1979; 41:124-128.
15. Kelly E. Changes caused by a mandibular removable partial denture opposing a maxillary complete denture. J Prosth. Dent 2003; 90:213-19.

16. Kamble S, Jankar A, Gajare S, Bangar B, Patil S, Somwanshi P. Combination syndrome: A Review Article. MIDSR J. Dent. Res.2020;2 :14-19.

17. Rutkunas V, Mizutani H, Peciuiliene V. Maxillary complete denture outcome with two-implant supported mandibular overdentures. A systematic review. Stomatologija 2008;10: 10-15

18. Tymstra N, Raghoebar G, Vissink A. Maxillary anterior and mandibular posterior residual ridge resorption in patients wearing a mandibular implant-retained overdenture. J. Oral Rehabil. 2011; 38:509-516.

19. Tiwari P, Karambelkar V, Patel J, Sethuraman R. Panoramic Radiographs for Evaluation of Maxillary and Mandibular Residual Ridge Resorption: In Vitro Study. J of Evolution of Med and Dent Sci.2014;60: 13380-13392.

20. Stuart C, Michael J. Oral Radiology Principles and interpretation. Chapter 11: Panoramic Imaging, 7TH edition, produced by Mosby Co., St. Louis, 2014.

21. Xie Q, Wolf J, Ainamo A., Quantitative assessment of vertical heights of maxillary and mandibular bones in panoramic radiographs of elderly dentate and edentulous subjects. Acta Odontol. Scand. 1997; 55:155-61.

22. Tallgren A. The continuing reduction of the residual alveolar ridges in complete denture wearers: a mixed-longitudinal study covering 25 years. J Prosth. Dent. 1972; 27:120-32.

23. Lopez-Roldan A., Abad D., Bertomeu I. Bone resorption processes in patients wearing overdentures. A 6-year retrospective study. Med Oral Patol. Oral Cir. Buccal 2009; 14:203-209 